\title{
Down-regulation of platelet adhesion receptors is a controlling mechanism of thrombosis, while also affecting post- transfusion efficacy of stored platelets
}

\author{
Ehteramolsadat Hosseini ${ }^{1}$, Maryam Mohtashami ${ }^{1}$ and Mehran Ghasemzadeh ${ }^{1,2^{*}}$ (D)
}

\begin{abstract}
Physiologically, upon platelet activation, uncontrolled propagation of thrombosis is prevented by regulating mechanisms which affect the expression and function of either platelet adhesion receptors or integrins. Receptor ectodomain shedding is an elective mechanism which is mainly involved in down-regulation of adhesion receptors GPIba and GPVI. Platelet integrin $a_{\| b} \beta_{3}$ can also be modulated with a calpain-dependent proteolytic cleavage. In addition, activating signals may induce the internalization of expressed receptors to selectively down-regulate their intensity. Alternatively, further activation of platelets is associated with microvesiculation as a none-selective mechanism which leads to the loss of membrane- bearing receptors. In a non-physiological condition, the storage of therapeutic platelets has also shown to be associated with the unwilling activation of platelets which triggers receptors down-regulation via aforementioned different mechanisms. Notably, herein the changes are timedependent and not controllable. While the expression and shedding of pro-inflammatory molecules can induce post-transfusion adverse effects, stored-dependent loss of adhesion receptors by ectodomain shedding or microvesiculation may attenuate post-transfusion adhesive functions of platelets causing their premature clearance from circulation. In its first part, the review presented here aims to describe the mechanisms involved in downregulation of platelet adhesion receptors. It then highlights the crucial role of ectodomain shedding and microvesiculation in the propagation of "platelet storage lesion" which may affect the post-transfusion efficacy of platelet components.
\end{abstract}

Keywords: $a_{\| b} \beta_{3}$, Adhesion, Ectodomain shedding, GPIba, GPVI, Microparticle, Platelet refractoriness, Transfusion, Thrombosis

\section{Highlights}

- Different mechanisms of internalization, microvesiculation and ectodomain shedding modulate platelet receptors

- Platelets lose the expression and function of adhesion receptors during storage

\footnotetext{
* Correspondence: mehran1476@yahoo.com

* Correspondence: mehran1476@yahoo.com
${ }^{1}$ Blood Transfusion Research Centre, High Institute for Research and

Education in Transfusion Medicine, Iranian Blood Transfusion Organization

Building, Hemmat Exp. Way, Next to the Milad Tower, PO Box: 14665-1157, Tehran, Iran

${ }^{2}$ Australian Center for Blood Diseases, Monash University, Melbourne, Victoria 3004, Australia
}

(c) The Author(s). 2019 Open Access This article is distributed under the terms of the Creative Commons Attribution 4.0 International License (http//creativecommons.org/licenses/by/40/) which permits unrestricted use distribution, and reproduction in any medium, provided you give appropriate credit to the original author(s) and the source, provide a link to the Creative Commons license, and indicate if changes were made. The Creative Commons Public Domain Dedication waiver (http://creativecommons.org/publicdomain/zero/1.0/) applies to the data made available in this article, unless otherwise stated.
- Ectodomain shedding and microvesiculation are mainly involved in platelet storage lesion (PSL)

- Platelet transfusion can be complicated by platelet refractoriness

- PSL decreases post-transfusion efficacy of platelets

\section{Introduction}

Platelets typically circulate in the bloodstream for 7-10 days and their principal function is to survey the inner lining of blood vessels to detect and seal any breaches in the vasculature by the creation of thrombi. The firm adhesion of platelets to the site of injury forms a monolayer which serves as a reactive site for further recruitment of free-flowing platelets. Platelet 
accumulation is dependent on platelet-bound Willebrand factor (vWF) and fibrinogen which serves to crosslink adjacent platelets and promote stable platelet aggregation $[1,2]$. This process is essential for the formation of a primary hemostatic plug (white thrombus) and also for the development of pathological thrombi at site of atherosclerotic plaque rupture [3]. Of note, in a developing thrombus further activation converts platelets from a proaggregatory to a to pro-coagulant phenotype which enables the assembly of the coagulation reaction complexes (the Tenase and Prothrombinase complex) on the cell surface, necessary for thrombin and fibrin generation. This supports secondary hemostasis by the conversion of the white thrombus to a red clot containing a planner of fibrin network and trapped RBCs [4]. Nonetheless, physiological thrombus formation (mural thrombi) is tightly regulated to avoid excessive platelet accumulation at the injury site and vascular obstruction, the principal pathological process causing heart attacks and ischaemic stroke [5]. Figure 1 demonstrates different steps of platelet tethering, adhesion, aggregation and thrombus formation.

\section{The important functions of platelet adhesion receptors}

Classically, thrombus formation on the site of vascular injury is triggered by the interaction of main platelets adhesive receptors Glycoprotein Ib/V/IX and Glycoprotein VI (GPVI) with their specific ligands which are exposed to circulation followed by endothelial damage. The initial capturing of free flowing platelets occurs through the binding of GPIb/V/IX to immobilized von vWF expressed at sites of vascular injury. This interaction slows down platelet movement and allows other adhesion receptors with slower-binding kinetics, including integrin $\alpha 2 \beta 1$ and GPVI, as the most potent adhesion receptor to be engaged with the exposed collagen in sub-endothelial matrix. Followed by GPVI ligation with collagen, the induction of strong inside-out signals induces platelet activation and release. These events are associated with integrin activation on the surface of both adhered and adjacent free flowing platelets while the interaction of these activated integrins with fibrinogen/ VWF can crosslink platelets to make aggregation and thrombus formation (Fig. 1a). Integrin ligation induce potent outside-in signals which augment cytosolic calcium influx associated with the conversion of proaggregatory phenotype of platelets located on developing thrombi to pro-inflammatory and pro-coagulant platelets [3]. P-selectin expressing platelets recruit leukocytes to the site of vascular injury while pro-coagulant platelets provide a highly efficient scaffold for coagulationcascade activity and fibrin generation which develop clot formation [6]. At this stage, polymerized fibrin has also shown to recruit circulating rest platelets lacking activated integrin through the interaction with adhesive receptors Glycoprotein Ib $\alpha$ (GPIb $\alpha$ ) and GPVI [7]. This second phase of platelet recruitments may provide a new scaffold for thrombin generation enhancing coagulant function. Developing thrombus also expresses a verity of pro-inflammatory mediators and receptors (Fig. 1b). Leukocytes recruitment to thrombi is initiated by the interaction between P-selectin and P-selectin glycoprotein ligand-1 (PSGL-1) which respectively are expressed on activated platelets and circulating rest leukocytes. Via this interaction, leukocyte begins to tether and roll around the thrombus while being affected by chemokines and chemoattractant which are continuously released from activated platelets. This cellular crosstalk induces potent inside-out signals which activate leukocyte integrin Machrophage-1 antigen (MAC-1). Followed by MAC-1 interaction with its main ligand in platelets, GPIb $\alpha$, leukocytes firmly adhere to thrombi while getting fully activated and polarized. These activated leukocytes may release chromatin neutrophil extracellular traps (NETs) which ensnare more circulating cells including platelets while providing an efficient negatively charged matrix that propagates pro-coagulant function [6, 8] (Fig. 1b).

\section{Different mechanisms modulating platelet adhesion receptors}

Changes of platelet receptors expression by different stimuli or during storage can influence their surface density and ligand binding capacity, signal transduction as well as receptor cross-linking and clustering. Regardless of platelets functional activity which can be affected by these alterations, physiologically, the changes may also control platelet aging or clearance. Studies have shown that the expression of adhesion receptors on the surface of platelets can be modulated by four different mechanisms including A) internalization, B) microvesiculation (microparticulation), C) secretion and D) ectodomain shedding. In addition, followed by ectodomain shedding, soluble forms of receptors lacking a transmembrane domain may also interfere with receptor functional activity while during microvesiculation, microparticle -bearing receptors may also play some important physio-pathological roles [9-11]. The controlled termination of signaling pathways after ligand-induced activation is an important mechanism to ensure appropriate signal intensity and the consequent cellular response [12]. Internalization, secretion and ectodomain shedding are three mechanisms involved in dampening ligation capacity and signal transduction via the selective removal of receptors from platelet membrane [13, 14]. These mechanisms which are ignited from early stage of platelet activation modulate different aspects of cellular 

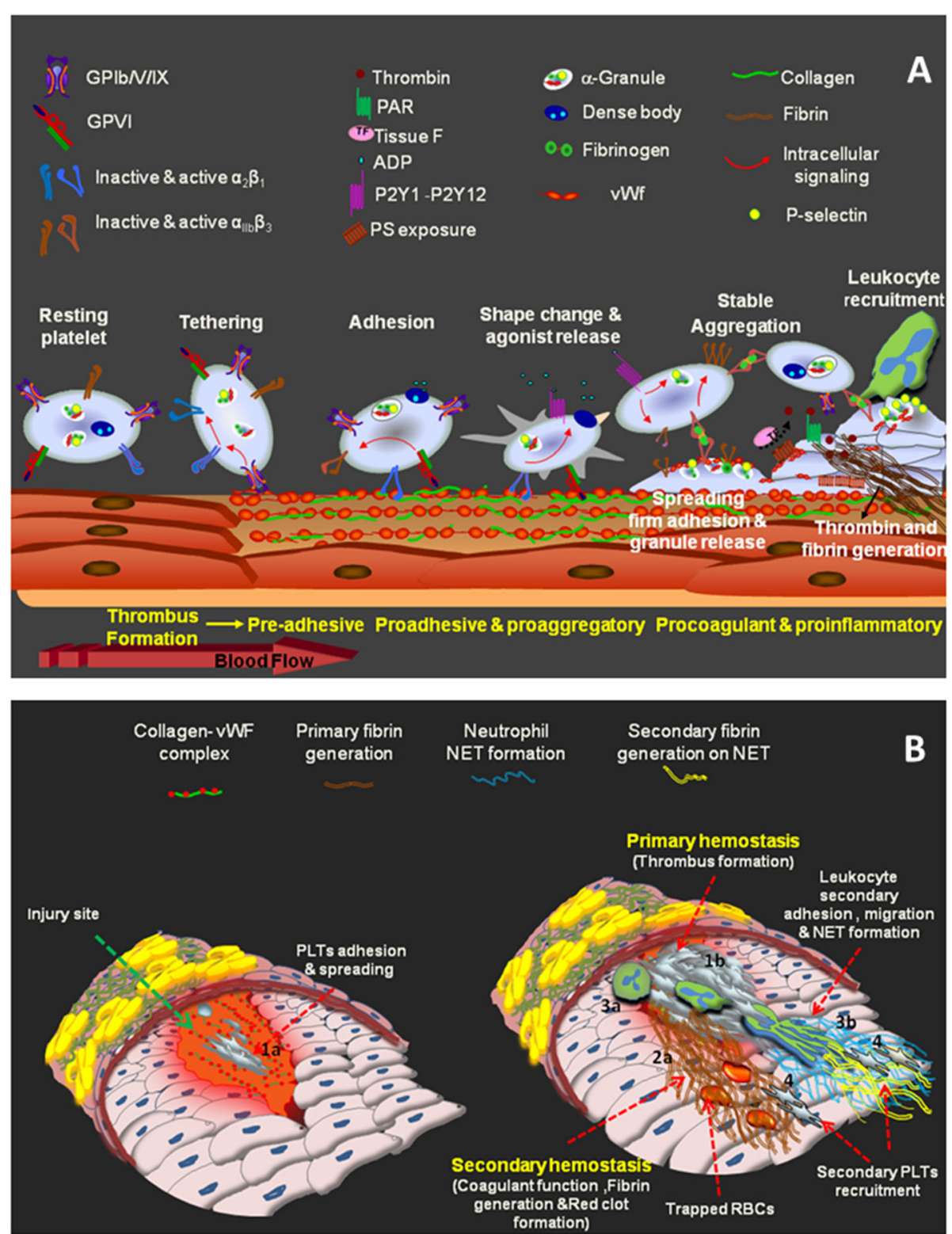

Fig. 1 a Different Stages of Platelet Adhesion to the Site of Vascular Injury. Resting platelets are initially captured on the site of vascular injury via the interaction between GPIb/N/IX and immobilized VWF (Tethering). Platelets firmly adhere to the sub-endothelial matrix through the engagement of collagen receptors a $2 \beta 1$ and GPVI (Adhesion). This triggers potent inside out signals inducing ADP release from dense bodies (Shape change \& agonist release) as well as activating platelet major integrin $a_{\| b} \beta_{3}$. Integrins facilitate platelet spreading and subsequent aggregation through the binding to VWF and fibrinogen. Activating signals down-stream engaged receptors induce the release of granule contents including P-selectin which provides an efficient scaffold for leukocyte recruitment linking pro-aggregatory phase of platelet activation to pro-inflammatory function. On the other hand, the accumulative signals further activate platelets and induce sustained calcium influx which results in the surface exposure of phosphatidylserine (PS) and pro-coagulant function leading to thrombin production and fibrin generation at the site of injury. Interacting with PAR receptors, generated thrombin also acts as a potent agonist which supports more efficient pro-

inflammatory and pro-coagulant function. b Primary and secondary hemostasis: mutual links between pro-inflammatory and pro-coagulant function 1- (a) Followed by the injury, platelet recruitment to the exposed sub-endothelial matrix leads to the formation of a developing thrombus(b) which express either pro-inflammatory molecules (mainly P-selectin) or pro-coagulant phospholipids (primary hemostasis). 2- Procoagulant matrix presented by platelets provides an efficient scaffold for fibrin generation (a) which supports secondary hemostasis by the conversion of the white thrombus to a red clot containing a planner of fibrin network and trapped RBCs.3- Platelets recruits leukocyte(a) while during their crosstalk, neutrophils get fully activated and release their chromatin contents as extracellular NET(b). The negatively charged NET materials provide an efficient pro-coagulant scaffold for fibrin generation. 4-Platelets may also interact with generated fibrin while creating a secondary thrombus 
functions including platelets trafficking and adhesion to the site of vascular injury as well as their proinflammatory and pro-coagulant functions during thrombus formation. Damaged receptors specially affected by oxidant stress have also shown to be eliminated by such selective removal mechanisms [15]. On the other hand, microvesiculation is considered as a none-selective mechanism of receptor removal, usually from fully activated platelets during membrane shedding or from apoptotic platelets by membrane fragmentation. Although platelets continuously shed microparticles (MPs) even under resting condition, significant microvesiculation occurs in post-activated or apoptotic end stage platelets [16].

\section{Internalization of platelet receptors}

Internalization can also affect platelet receptors redistribution by different specific and nonspecific mechanisms. One of these mechanisms is receptor translocation into the open canalicular system (OCS). This is an important mechanism to regulate the redistribution of GPIb and GPIV [17] . GPVI, platelet G-protein coupled receptors (PAR-1, PAFR, $\left.P_{2} Y_{1}, P_{2} Y_{12}\right)$, integrins $\left(\alpha_{2} \beta_{1}, \alpha_{I I b} \beta_{3}\right)$, Platelet-derived growth factor receptor $\alpha$, thrombopoietin receptors and prostacyclin receptors are also other platelet receptors which are internalized by different mechanism [18-24].

\section{General mechanisms of receptor internalization}

Upon activation, internalization redistributes the receptors while shuttling them from the plasma membrane to endosomes where they may experience inactivation process through lysosomal degradation [25, 26]. Generally, endocytosis is triggered by ubiquitin molecules interaction with activated receptors and their related proteins which are associated with tyrosine kinase dependent Phosphorylation [12]. One of the important components of endocytic machinery is clathrin which is likely regulated by ubiquitination processes [27]. When a clathrin-coated pit forms on the inner surface of the cell membrane, it then buds inside the cell and forms a coated vesicle containing receptors and other membrane associated molecules. Cytoplasmic proteins including dynamin and adaptors such as adaptin may be also involved in endocytosis process [28]. Other molecular factors which may affect membrane receptors redistribution are included in cytoskeletal proteins and lipid composition [29].

Receptor endocytosis may also occur constitutively without cellular activation. This is a clathrin-independent pathway of endocytosis which is not affected by receptor antagonisms or associated with receptor signaling [26]. In some condition receptor redistribution may be induced even by non-molecular factors such as external physical stress which affects membrane integrity and cause none specific endocytosis [29].

\section{Platelet Microvesiculation}

Notably, since more than $80 \%$ of circulating microparticles express platelet antigens in healthy individuals, the physiological importance of platelet-originated microparticles and their relevance with the status of different diseases especially cardiovascular and inflammatory disorders, including arterial thrombosis, heparin-induced thrombocytopenia, immune thrombocytopenia, malaria infection, acquired immune deficiency syndrome (AIDS), and rheumatoid arthritis have been so far of interests in numerous researches $[30,31]$. Platelets release two types of vesicles including exosomes (approximately 40-100 $\mathrm{nm}$ in diameter) which are shed by exocytosis from the multivesicular bodies as well as alpha-granules and microparticles (approximately 100-1000 $\mathrm{nm}$ in diameter) that are released by budding of the cytoplasmic membrane or by platelet fragmentation during apoptosis [10]. As a general marker, like other type of microparticles, platelet derived microparticles; (PMPs) usually express Annexin-V or tissue factor (TF). PMPs also express variety of platelet surface molecules including adhesive receptors (such as GPIb $\alpha, \beta_{3}$ integrin (GPIIIa, CD61), $\alpha_{\mathrm{IIb}}{ }^{-}$ integrin (GPIIb, CD41), GPVI, P-selectin, lysosomalassociated membrane protein3 (LAMP3, CD63), thrombospondin -1 and Platelet endothelial cell adhesion molecule (PECAM)-1 [31, 32]. Therefore, membrane loss during microparticulation is associated with the loss of platelet surface proteins including adhesive receptors. Given this, microparticle formation can be considered as a down-regulating mechanism of platelet adhesiveness to reactive matrixes. However, PMPs show diverse phenotypes based on their activation states. Resting plateletsderived microparticles are phosphatidylserine (PS) negative, which not expressing pro-inflammatory molecules including P-selectin whereas activated platelets express PS, P-selectin and CD40 ligand (CD40L) [33]. In addition, as an another important pro-inflammatory molecule, PMPs also showed to express plateletactivating factor (PAF) [34]. Amongst adhesion molecules expressed by microparticle, GPVI also detected on megakaryocyte microparticles while not being expressed by microparticles released from activated platelets [35]. This may suggest that, contrary to other adhesion receptor GPIb $\alpha$ (which is significantly expressed by PMPs), GPVI down regulation on platelets cannot be affected by microparticulation and mainly modulated by other mechanisms including ectodomain shedding or internalization. Notably, regardless of expressed molecules, PMPs also comprise a vast array of biologic materials including growth factors, coagulation factors, enzymes, chemokines, cytokines, complement proteins, apoptosis 
regulators, bioactive lipids, miRNAs, $\beta$-amyloid precursor protein, $\mathrm{Ca}^{2+}$-dependent protease) calpain (, which may play important roles in different biological functions [36].

\section{Microvesiculation mechanism in platelets}

Membrane integrity in resting platelets can be affected with intensive physical stress induced by some pathological states such as the presence of abnormal shear force in atherosclerotic vessels, mechanical artificial valve and microangiopathic conditions [37]. Contrary to agonist-induced microparticle generation or what occurs in activated platelets, Destabilization of the actin cytoskeleton in resting platelets can trigger microparticle shedding by the mechanisms which are not dependent to $\mathrm{Ca}^{2+}$ elevation and calpain activity [30]. However, the involvement of the phosphoinositide 3-kinase signaling pathway in the articulation of $\alpha_{\mathrm{II}} \beta_{3}$-integrin with actin dynamics might be one of the possible mechanisms [38]. Upon platelet activation, the elevation of intracellular $\mathrm{Ca}^{2+}$ and ROS generation (through cyclooxygenase and $\mathrm{NADPH}$ oxidase) induce calpains activation and cytoskeletal changes. Clapains cleave skeleton associated proteins in platelet membrane while aberrantly destabilizing actin polymerization and cytoskeletal arrangement $[39,40]$. Generally, Calpain activity in addition to the loss of membrane integrity induced by PS exposure, are the main mechanisms behind microparticle shedding in activated platelets. Moreover, shear stress-induced PMPs formation may also occur during platelet activation and adhesion through the interaction between GPIb $\alpha$ and vWf where the loosely adhered platelets are tethering off from the adhesive matrix. This means that the circulating detached platelets lose some parts of their membrane contents including adhesive receptors [41]. With fairly different mechanisms involved, platelet apoptosis is also associated with the significant microparticle formation during membrane fragmentation which might be mechanistically different from calcium dependent budding of cell membrane in activated platelets. Platelet apoptosis is associated with mitochondrial damage, cytochrome release and caspase activity which leads to both calpain dependent and independent cytoskeleton cleavage/reorganization resulting in microparticles release from remnant of the apoptotic cell $[16,42]$. Activation based receptor modulation has been also shown to be relevant with microparticle formation. In addition to calpain, GPIb $\alpha$ modulation during platelet activation may also affect microvesiculation in stored platelets. A current study has indicated that upon platelet activation, cytosolic phospholipase A2 (PLA2) activity with subsequent AA production can result in dimerization of 14-

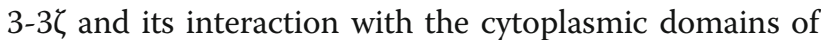
GPIb $\alpha$ in lipid rafts. While this interaction induces the stabilization and clustering of GPIb $\alpha$, on the other hand, it causes the loss of the membrane anchoring interaction of Filamin A with this receptor, which affects platelet surface integrity and makes the membrane prone to microvesiculation [43].

\section{Ectodomain shedding in platelets}

Ectodomain shedding through limited proteolysis is a general mechanism to modulate the function of surface transmembrane proteins. This process usually occurs during cell activation and is associated with the proteolysis of a broad range of transmembrane macromolecules including adhesion receptors, numerous cytokines, growth factors and their receptors. However, resting (non-stimulated) cells also experience a basal level of constitutive ectodomain shedding which is increased in a time-dependent manner, while can be dramatically enhanced in response to activating stimuli or apoptotic conditions $[44,45]$. Platelet adhesion and activation has been shown to be associated with surface protein shedding [46] and since platelets have not the translational capability to renew their full complement of surface proteins, ectodomain shedding is considered to be a rapid and irreversible key mechanism which effectively downregulates the surface expression of platelet receptors and their signaling capacities [11, 13]. Endogenous platelet metalloproteinases (sheddases) including a disintegrin and metalloproteases (ADAMs) and matrix metalloproteinases (MMPs) are the key players in platelet ectodomain shedding [47]. Ectodomain shedding has been reported as an important mechanism for the regulation of GPIb $\alpha$ and GPV expressions on the platelet surface. In addition to down-regulation of the interaction between GPIb-V-IX and vWF, this phenomenon has been also suggested to be responsible for the clearance of aged platelets from the circulation [48]. Actually desialylation of GPIb-V-IX through sialidase activity also causes platelet clearance while inducing the irreversible formation of receptor clustering and the ectodomain shedding of GPIb $\alpha$ and GPV from the platelets [49, 50]. ADAM17 is the most prominent endogenous sheddas, which cleaves both GPIba and GPV at specific sites releasing a 130- and an 80-kDa soluble fragment, respectively [48]. The $130 \mathrm{kDa}$ fragment is referred as glycocalicin (GC) and circulates in plasma at a concentration of 1 to $3 \mu \mathrm{g} /$ $\mathrm{mL}$ [51] . Generally, remarkable function of ADAM 17 occurs during platelet activation by a variety of agonists, however due to the presence of constitutive levels of mature ADAM17, even resting platelets experience basal levels of GPIba and GPV shedding. Evidence for the physiological regulation of GPIb $\alpha$ by ADAM 17 can be derived from the observation that the concentration of glycocalicin in the plasma of the mice deficient in functional ADAM17 is considerably lower than wild type 
animals [52]. In addition to cell activation, GPIbo shedding also occurs during cell death. Treatment of platelets with carbonyl cyanide 3-chlorophenylhydrazone (CCCP) provokes mitochondrial injury and platelet apoptosis similar to that observed during the storage of platelets in vitro. In both scenarios, ADAM17 has been suggested as the responsible protease $[45,52,53]$. As the main collagen receptor, GPVI is another molecule which is shed from the platelet surface during platelet activation by agonists as well as shear stress. However, these shedding events are mainly modulated by ADAM10 while leading to the release of a soluble $\sim 55-\mathrm{kDa}$ fragment with a $\sim$ $10 \mathrm{kDa}$ platelet-associated remnant fragment. In addition, similar to GPIb $\alpha$, the mitochondrial damage also shown to be associated with GPVI shedding in a mechanism which is mainly ADAM 17 dependent [54]. Resting platelets also constitutively present both ADAM 10 and ADAM 17. However contrary to what already mentioned for GPIb and despite the presence of ADAM10 with detectable proteolytic activity on the surface of resting platelets, GPVI expression has shown to be quite stable on circulating inactivated platelets. This might be due to cryptic nature of vulnerable site of GPVI which only expose to ADAM 10 proteolytic activity under the condition of ligand binding or membrane perturbation in elevated fluid shear stress [55].

\section{Mechanistic features for the shedding of platelet adhesion receptors}

Several lines of evidence suggested that ADAMdependent shedding of platelet adhesion receptors can be modulated by $\mathrm{Ca}^{2+}$ elevation, protein kinase $\mathrm{C}$ (PKC) activation, PS exposure, and caspase activity [56]. A calcium-modulated protein, calmodulin is one of the important modulators of ectodomain shedding. It has been shown that the direct attachment of calmodulin to juxtamembrane cytoplasmic sequences of GPV, GPIb $\beta$ (from GPIb-V-IX complex) and GPVI receptor can interfere with the activity of the sheddase. This is supported by the observation that dissociation of calmodulin from the ectodomain cleavage sites, induced by calmodulin inhibitors such as W7, renders receptors more vulnerable to shedding. It has been shown that calmodulin disassociation from these inhibitors significantly increases the levels of sGPVI and GC. On the other hand, signaling events followed by platelet activation can increase intracellular calcium while its interaction with calmodulin can be associated with rapid conformational changes of the molecule and its dissociation from receptor cleavage sites which make them vulnerable to the proteolytic activities by ADAMs [57]. The another possibility is that the interaction between those affected calmodulin and some cytoskeleton modulators such as myosin light chain kinase (MLCK) causes cytoskeletal rearrangement which may induce shedding event by the effects on either receptors stability or ADAMs activity [40, 57, 58]. Additionally, calmodulin may affect receptor shedding through its involvement in other pathways including calcium/calmodulin-dependent protein kinase type I/II (CaMKI/II) while calmodulin antagonists also showed to induce receptor shedding by the activation of apoptotic pathways or calcium elevation $[59,60]$. Generally, platelet activation and $\mathrm{Ca}^{2+}$ elevation is associated with increased levels of intracellular reactive oxygen species (ROS) [61]. ROS-induced oxidation of cysteine residues located on cysteine-rich domain of ADAMs can activate these proteolytic molecules, while ROS interaction with intracellular cytoplasmic domains of ADAMs may also increase their affinity with their substrate [58]. On the other hand, the direct or indirect interaction of ROS with cytoplasmic domains of the adhesive receptors GPVI may also induce shedding events. It has been indicated that following ligand binding of GPVI, rapid oxidation of an unpaired thiol in the cytoplasmic tail of this receptor results in the formation of GPVI homodimers, preceding down-stream signaling and ectodomain metalloproteolysis of GPVI $[62,63]$. ROS also can indirectly affect the receptors and induce their shedding by the oxidation of cysteine residues of different related protein kinases including p38-MAPK (mitogen-activated protein kinase), PTP (protein tyrosine phosphatase), SFK (sarcoma family kinase) and PKs C, G, or A [64-66]. It is known that ADAM17 (as the main sheddas of platelet GPIb $\alpha$ and GPV) is activated via a p38 MAPKdependent pathway which is per se modulated by accumulating ROS [67]. On the other hand, activated p38MAPK can lead to the dimerization of the protein 14,3, $3 \zeta$ attached to the cytoplasmic domain of GPIb $\alpha$. This induces the receptor clustering and its disassociation from filamin which makes it unstable and susceptible to shedding events [40]. ROS-induced activation of p38MAPK, PKC and SFK can enhance NADPH oxidase activity leading to further ROS generation [68]. The generated ROS can activate other signaling pathways such as PLC and PI3K which increase intracellular calcium by its either release from platelet DTS or entrance by specific channels [61]. Consequently, this ROS-induced calcium influx can enhance receptor shedding in the way already discussed.

\section{Proteolytic modulation of platelet receptor integrin $\alpha_{1 \mathrm{lb}} \beta_{3}$}

It has been currently shown that platelets can experience the ectodimain shedding of $\alpha_{\mathrm{II}} \beta_{3}$ under nonphysiological shear stress while the concentration of this receptor components has been increased in microparticle-free plasma [69]. However, more studies are required to characterize a specific sheddase for $\alpha_{\mathrm{IIb}} \beta_{3}$ 
or define an in vivo pathophysiologic importance for this observation and its relevance to the mechanisms of integrin down-regulation. Additionally, a recent study also claimed that oxidative stress can induced modulation of platelet integrin $\alpha_{\mathrm{II}} \beta_{3}$ expression and shedding in patients implanted with continuous flow left ventricular assist devices (CF-LVADs). However, in this study samples examined for the receptor shedding analysis were not microparticle-free and since PMPs are rich of $\alpha_{\mathrm{II}} \beta_{3}$, both observed reduction in the expression of $\alpha_{\mathrm{IIb}} \beta_{3}$ and higher levels of soluble integrin could be resulted from platelet microvesiculation rather than ectodomain shedding [70]. Regardless of these reports, so far all other studies have not reported ectodomain shedding for $\alpha_{I I b} \beta_{3}$ in any in vivo or in vitro experimental models. On the other hand, In different condition of either agonistinduced or storage-dependent PMPs formations, our western blot analysis also confirmed that using multisteps ultra-centrifugation can totally reduce the soluble levels of $\alpha_{\mathrm{IIb}} \beta_{3}$ in microparticle-free samples [54] . However, studies showed that, following platelet activation especially within sustained calcium influx, calpaindependent proteolytic cleavage of cytoplasmic part of $\beta 3$ may affect $\alpha_{\mathrm{IIb}} \beta_{3}$ activation and clustering [48] .Several line of evidence have also reported calpain-mediated cleavage of integrin-dependent signaling molecules (including FAK, Src, PTP-1B) and some related cytoskeletal proteins (such as talin, filamin-1, and cortactin). This may also be considered as an alternative proteolytic pathway which can control the adhesive function of $\alpha_{\mathrm{II}} \beta_{3}$ while regulating thrombus formation. However, the role of calpain in regulating integrin activity still remains controversial as shown that this protease may present either positive or negative effects on function of $\alpha_{\text {IIb }} \beta_{3}$ platelets [71].

\section{Platelet receptors crosstalk and ectodomain shedding}

Receptors crosstalk is also considered as another feature of shedding mechanism. The studies showed that the GPIb ligation causes its cross-interaction with FC receptors (FCR) $\gamma$ and FCRIIa through the PI3K signaling pathway [72]. The interactions induce signaling cascades down-streams of these two receptors leading to the enhancement of GPVI shedding [73]. C-type lectin-like receptor 2 (CLEC-2) is a currently described molecules expressed by platelets, which can interact with Podoplanin as a widely expressed molecule outside of the vasculature while inducing activating signals through a hemimmunoreceptor tyrosine-based activation motif (hemITAM). It has been shown that the ligation of this receptor is associated with the proteolytic cleavage of GPVI and FcgRIIa. However, there is no evidence that shows CLEC-2 shedding during platelet activation [31]
.Integrin involvement in ADAMs activation is another calcium-dependent mechanism which can control shedding events. It is known that followed by inside-out signaling, integrin gets primed while its ligation with fibrinogen can induce significant activating outside-in signals which ignite calcium influx, ROS generation and consequent receptors shedding [61, 74]. Still, there is one more intriguing mechanism by which integrin can modulate shedding through its direct interaction with ADAMs. In one side, integrin binding to the ADAMs can activate these molecules enhancing the shedding of adhesive receptors while this interaction also can modulate integrin ligation capacities attenuating outside-in signaling [75]. It seems that, this mechanism can efficiently down-regulate platelets adhesive function through the either decrease in platelet activation or increase of receptor shedding. On Receptor shedding could also be essentially $\mathrm{Ca}^{2+}$ independent. There are some PKC mimetic compounds, such as phorbolmyristate-acetat (PMA) which act independent of $\mathrm{Ca}^{2+}$ while significantly increasing ADAMs activities and shedding [56, 76].

\section{Platelet apoptosis and receptor shedding}

Platelet apoptosis is associated with the significant ectodomain shedding of surface receptors. Ex vivo studies showed that the treatment of human platelets with BH3 mimetic agents can decrease platelet adhesive function due to ectodomain shedding of GPIb $\alpha$ and GPVI. On the other hand, thrombocytopenic mice deficient in Bcl$\mathrm{xL}$ also showed the reduced expression of GPIb $\alpha$ and GPVI in platelets [46, 77], confirming the important role of apoptotic pathway in shedding process. In addition to BH3 mimetic agents that induce a time- and dosedependent decrease in platelet adhesive function resulted from ectodomain shedding of GPIb $\alpha$ and GPVI, the mitochondrial poison carbonyl cyanide 3chlorophenylhydrazone (CCCP) also creates premature platelet ageing and shedding of these platelet receptors [78]. Although, apoptosis-induced shedding of receptors is mainly independent of $\mathrm{Ca} 2+$, its exact mechanism is not well characterized yet, as $\mathrm{BH} 3$ mimetic agents like ABT 737 also showed to induce a small increases in $\mathrm{Ca} 2+$ in a minor platelet population [79]. It has been shown that under apoptotic condition, metalloproteinase inhibitors can significantly reduce receptor shedding. Therefore, it is postulated that the activation of metalloproteinase downstream of mitochondrial injury and caspase activation can be the main event that regulates receptor shedding under apoptotic condition. Studies showed that target receptor shedding by activated ADAM17 is modulated in a p38-dependent manner while the inhibition of p38-MAPK can block the shedding of GPIb $\alpha$ in stored platelets [46]. On the other 
hand, studies also confirmed that p38-MAPK significantly plays a role in ABT 737-induced platelet apoptosis; however whether the activation of this kinase during apoptosis can be also involved in platelet receptor shedding requires further investigation [80]. Of note, in apoptotic condition, the possible mechanism by which MAP kinase regulating ADAMs activity and shedding cannot be ROS-dependent as already described under activation status because $\mathrm{BH} 3$ mimetic agents did not show to whether induce or increase intra platelet ROS generation [80].

\section{Physiological significance for the shedding of platelet adhesive receptors}

In a physiological basis, shedding of adhesion receptors is considered as part of a mechanistic process facilitating platelet spreading via the sequential events of platelet de-attachment and re-attachment at the tips of filopodia or the leading edge of lamellipodia. In addition receptor shedding is an important mechanism modulating thrombotic function through a controlled down-regulation of platelets reactivity [48]. However, in some cases, the uncontrolled shedding of adhesion receptors can molest platelet function and hemostasis [81]. Lowered surface density of GPIb $\alpha$ and GPVI due to the shedding can attenuate signaling capacity which affects platelet activation and secretion. This is in addition to the platelet adhesive malfunction which is in a direct correlation with lower expression of these receptors. On the other hand, ectodomain shedding of adhesion receptors can also decrease the number of platelet-matrix contacts, which potentiates thrombo-embolic events [48]. It has been shown that in thrombi formed ex vivo, procoagulant platelets undergoing ADAM-induced glycoprotein shedding are less-adhesive due to defects in GPIb $\alpha$ and GPVI dependent adhesion to VWF, collagen, and fibrin. In addition calpain-induced integrin cleavage in pro-coagulant platelets can be also considered as another reason aggravating platelet signaling capacity and adhesive capabilities [56]. Notably, pro-coagulant function is associated with the excessive microparticle formation leading to the loss of platelet membrane containing adhesion receptors [81]. Thus in a same scenario but with a different mechanism, platelets lose more adhesive capacities within post activation stage.

\section{Platelet storage and increasing loss of adhesion receptors}

Transfusion of platelet concentrates (PCs) is one of the most important therapeutic approaches in the management of thrombocytopenia and bleeding complications. Regardless of preparation techniques, stored platelets gradually experience inevitable deleterious changes socalled platelet storage lesion (PSL) that may lead to a progressive structural and functional damage. These changes which begin from the time of platelets isolation till the transfusion to a recipient are mainly induced by either reversible or irreversible increases in the basal levels of platelet activation. The most important phase of PSL is associated with irreversible changes in platelet morphology and function, which are significantly initiated with platelet $\alpha$ granules release and its conversion from pro-aggregatory status to pro-inflammatory phenotypes identified by the expression of $\mathrm{P}$-selectin and CD40L [54]. It has been shown that along with platelet activation, the increased levels of intracellular calcium acts as the main modulator of signaling events which results in platelet receptor ectodomain shedding and membrane loss due to microparticulation. Both of these phenomena cause progressive loss of adhesive receptors during platelet storage [82]. Notably this extensive loss of platelet receptors may affect its proper function required for therapeutic uses [54, 83]. Decreasing levels of GPIb $\alpha$ expression during the storage of platelet concentrates (PCs) were reported by several studies [54]. Receptor ectodomain shedding and platelet membrane loss due to microparticulation are two important causes for this gradual decrease [82]. We already indicated that the continuous shedding can lead to significant decrease in GPIb $\alpha$ expression in 5-day stored platelets [54]. It is postulated that the down-regulation of GPIb $\alpha$ expression affect the adhesive function of transfused platelet in elevated arterial shear rates [84]. Obviously the older platelet products with the lower GPIb $\alpha$ expression may show less functionality after transfusion. This is in addition to the fact that metalloproteinase- dependent loss of surface GPIb $\alpha$ plays a role in the clearance of aged platelets from the circulation [52]. Therefore, the higher levels of shedding for this receptor in older PCs could be also associated with a rapid clearance of transfused platelets leading to reduced platelet recovery and survival. GPVI expression also showed to be modulated during storage. We already demonstrated a decreasing expression of GPVI during storage where was directly correlated with the GPIb $\alpha$ expression. A negative correlation between GPVI expression and shedding was also observed, a finding that verifies the main role of ectodomain shedding in the modulation of GPVI expression. It is worthy to know that given the absence of GPVI in the proteome of platelet microparticles, dislike GPIba, the reducing levels of GPVI expression should be mainly due to ectodomain shedding rather than microvesiculation. A direct correlation between the shedding levels of P-selectin and GPVI during storage suggested this adhesion receptor as a valid marker of PSL [54] . This finding was also consistent with data from clinical studies in which the levels of soluble GPVI was correlated with soluble P-selectin in patients with acute coronary syndrome and or with 
Acute Ischemic Stroke [85]. Further studies also suggested soluble GPVI as a helpful biomarker to monitor platelet activation during anti-platelet therapy [86]. Notably considerable shedding of GPVI and its association with decreasing expression of this receptor on the surface of stored platelets can affect GPVI dependent platelet function during storage. Some studies showed significant attenuation in platelet adhesion capacity to collagen in stored PCs $[87,88]$. We also showed a direct correlation between this observed decreasing adhesive capacities with the increasing levels of GPVI shedding during storage. However, whether the observed loss of GPVI during storage can render platelet signaling capacities and integrin activation was another important question which has been addressed by the observation of attenuating platelet aggregation in responses to collagen during storage and its significant reverse correlation with GPVI shedding in stored PCs [89] .

\section{Clinical significance of PSL}

Although clinical research has not yet found any significant correlation between PSL and post-transfusion adverse effects or bleeding events in the patients, these studies have confirmed that storage duration can affect platelets survival in circulation. It is known that transfusion of fresher platelets is accompanied with higher CCI (corrected count increment) and more prolonged transfusion intervals in patients [90]. In other word, clinically, transfusion of the longer stored PCs has been associated with reduced CCI, platelet recovery and survival [91]. This might be due to the fact that in longer stored products, platelets with P-selectin exposure, GPIb $\alpha$ shedding and reduced membrane-associated sialic acid are selectively cleared from circulation [87, 92] .Using the mice models, in vivo studies showed that in stored platelets the inhibition of GPIb $\alpha$ shedding by metalloproteinase inhibitor or 5G6 Fab fragment can promote post transfusion recovery of platelets while improving their hemostatic function [87, 93]. Conclusively these studies suggest that GPIb $\alpha$ shedding reduces platelet reactivity while increasing its clearance in vivo. However, while lower CCI, platelet recovery, clearance and survival are much related to expression levels of adhesion receptors has not well defied yet.

\section{Conclusion}

Therapeutic platelet transfusion can be complicated by platelet refractoriness either modulated by immune or non-immune causes. Immune-related refractoriness especially those induced by antibodies against HLA antigens are the most studied causes of post transfusion platelet malfunctions. However platelet storage dependent lesion seems to play an important role in the premature clearance of transfused circulating platelets, which also results in lower efficacy for platelet transfusion. Evidently platelet storage is associated with the ectodomain shedding and microvesiculation which down-regulate platelet adhesion receptors, GPIb $\alpha$ and GPVI. This is in addition to the storage-dependent loss of integrin activity, which is also crucially involved in platelet aggregability and thrombus formation. Whereas these alterations have shown to irreversibly affect platelet function in vitro, clinical observations have indicated that the premature clearance or lower survivals rather than platelet malfunction is the only important adverse effect of platelet transfusion. Therefore given the improvement of hemostasis in the patients who transfused the longer-stored platelets, some studies suggested that platelets dysfunction due to PSL is generally restored after transfusion. However, this is a controversial viewpoint, as basic studies have confirmed that platelet adhesion during storage is affected by irreversible downregulating mechanisms such as ectodomain shedding, micopaticulation and other proteolytic cleavage which permanently destroy some receptor adhesive and signaling function. Thereby highlighting detailed mechanisms involved in down-regulation of platelet adhesion receptor,This review may benefit the readership to better judge whether the functional capabilities of stored platelets can be properly recovered after transfusion or not.

\section{Abbreviations \\ ADAMs: A disintegrin and metalloproteases; AIDS: Acquired immune deficiency syndrome; CaMKI/II: Calcium/calmodulin-dependent protein kinase type I/ll; CCCP: Carbonyl cyanide 3-chlorophenylhydrazone; CCl: Corrected count increment; CD40L: CD40 ligand; CF-LVADs: Continuous flow left ventricular assist devices; CLEC-2: C-type lectin-like receptor 2; FCR: FC receptors; GC: Glycocalicin; GPIba: Glycoprotein Iba; GPVI: Glycoprotein Vl; hemITAM: Hemimmunoreceptor tyrosine-based activation motif; LAMP3: Lysosomal-associated membrane protein 3; MAC-1: Machrophage-1 antigen; MAPK: Mitogen-activated protein kinase; MMPs: Matrix metalloproteinases; MPs: Microparticles; MLCK: Myosin light chain kinase; NETs: Neutrophil extracellular traps; OCS: Open canalicular system; PI3K: Phosphoinositide 3-kinases; PMA: Phorbol-myristate-acetat; PS: Phosphatidylserine; PLA2: Phospholipase A2; PAF: Platelet activating factor; PCs: Platelet concentrates; PECAM: Platelet endothelial cell adhesion molecule; PMPs: Platelet derived microparticles; PSL: Platelet storage lesion; PKC: Protein kinase C; PSGL-1: P-selectin glycoprotein ligand-1; PTP: Protein tyrosine phosphatase; ROS: Reactive oxygen species; SFK: Sarcoma family kinase; TF: Tissue factor; VWF: Von Willebrand factor}

\section{Acknowledgements}

A few parts of this review are extracted from MG's doctorate thesis at Monash University (as referred). The authors wish to thank Prof. Shaun P. Jackson from Australian center for blood diseases for his constructive advice.

\section{Authors' contributions}

MG provided intellectual input designed and wrote the paper as main author, depicted the figures. EH provided intellectual input, co-wrote the paper and depicted the figures. MM provided intellectual input and helped with the writing of paper. All authors read and approved the final manuscript.

Funding

No funding evolved. 


\section{Availability of data and materials}

Data sharing not applicable to this article as no datasets were generated or analysed during the current study.

\section{Ethics approval and consent to participate} Not applicable.

\section{Consent for publication}

Not applicable.

\section{Competing interests}

The authors declare that they have no competing interests.

Received: 18 July 2019 Accepted: 10 September 2019

Published online: 23 October 2019

\section{References}

1. Kulkarni S, Dopheide SM, Yap CL, Ravanat C, Freund M, Mangin P, Heel KA, Street A, Harper IS, Lanza F, Jackson SP. A revised model of platelet aggregation. J Clin Invest. 2000;105(6):783-91.

2. Ruggeri ZM, factor $\vee$ W. J Clin Invest. 1997;100(11 Suppl):S41-6.

3. Hosseini E, Ghasemzadeh M. Different stages of platelet adhesion to the site of vascular injury. Iranian J Blood Cancer. 2012;4(3):133-42.

4. Investigation of Signaling Cross-talk Between Platelets and Neutrophils. Melbourne: Monash University; 2009.

5. Gibbins JM. Platelet adhesion signalling and the regulation of thrombus formation. J Cell Sci. 2004;117(16):3415-25.

6. Ghasemzadeh M, Hosseini E. Platelet-leukocyte crosstalk: linking proinflammatory responses to procoagulant state. Thromb Res. 2013;131(3): $191-7$.

7. Mammadova-Bach E, Ollivier V, Loyau S, Schaff M, Dumont B, Favier R, Freyburger G, Latger-Cannard V, Nieswandt B, Gachet C, Mangin PH, Jandrot-Perrus M. Platelet glycoprotein VI binds to polymerized fibrin and promotes thrombin generation. Blood. 2015;126(5):683-91.

8. Ghasemzadeh M, Hosseini E. Intravascular leukocyte migration through platelet thrombi: directing leukocytes to sites of vascular injury. Thromb Haemost. 2015;113(06):1224-35

9. Gardiner EE, Al-Tamimi M, Andrews RK, Berndt MC. Platelet receptor shedding. Methods Mol Biol. 2012;788:321-39.

10. Melki I, Tessandier N, Zufferey A, Boilard E. Platelet microvesicles in health and disease. Platelets. 2017;28(3):214-21.

11. Chen Z, Mondal NK, Ding J, Koenig SC, Slaughter MS, Griffith BP, Wu ZJ. Activation and shedding of platelet glycoprotein IIb/llla under nonphysiological shear stress. Mol Cell Biochem. 2015;409(1-2):93-101.

12. Holler D, Dikic I. Receptor endocytosis via ubiquitin-dependent and -independent pathways. Biochem Pharmacol. 2004;67(6):1013-7.

13. Hayashida K, Bartlett AH, Chen Y, Park PW. Molecular and cellular mechanisms of ectodomain shedding. Anat Rec (Hoboken). 2010;293(6): 925-37.

14. Watanabe S, Boucrot E. Fast and ultrafast endocytosis. Curr Opin Cell Biol. 2017:47:64-71.

15. Piper RC, Lehner PJ. Endosomal transport via ubiquitination. Trends Cell Biol. 2011;21(11):647-55.

16. Flaumenhaft R. Formation and fate of platelet microparticles. Blood Cells Mol Dis. 2006;36(2):182-7.

17. Lopez-Vilchez I, Hedner U, Altisent C, Diaz-Ricart M, Escolar G, Galan AM Redistribution and hemostatic action of recombinant activated factor VII associated with platelets. Am J Pathol. 2011:178(6):2938-48.

18. Wang Z, Leisner TM, Parise LV. Platelet a $2 \beta 1$ integrin activation: contribution of ligand internalization and the a2-cytoplasmic domain. Blood. 2003;102(4): 1307-15.

19. Smith TH, Li JG, Dores MR, Trejo J. Protease-activated receptor-4 and purinergic receptor $\mathrm{P} 2 \mathrm{Y} 12$ dimerize, co-internalize, and activate Akt signaling via endosomal recruitment of beta-arrestin. J Biol Chem. 2017; 292(33):13867-78

20. Dupre DJ, Chen Z, Le Gouill C, Theriault C, Parent JL, Rola-Pleszczynski M, Stankova J. Trafficking, ubiquitination, and down-regulation of the human platelet-activating factor receptor. J Biol Chem. 2003;278(48):48228-35.

21. Avrov K, Kazlauskas A. The role of c-Src in platelet-derived growth factor a receptor internalization. Exp Cell Res. 2003;291(2):426-34.
22. Rabie T, Strehl A, Ludwig A, Nieswandt B. Evidence for a role of ADAM17 (TACE) in the regulation of platelet glycoprotein V. J Biol Chem. 2005; 280(15):14462-8

23. Midgett C, Stitham J, Martin KA, Hwa J. Prostacyclin receptor regulationfrom transcription to trafficking. Curr Mol Med. 2011;11(7):517-27.

24. Gao W, Shi P, Chen X, Zhang L, Liu J, Fan X, Luo X. Clathrin-mediated integrin alphallbbeta3 trafficking controls platelet spreading. Platelets. 2018; 29(6):610-21.

25. Koenig JA. Assessment of receptor internalization and recycling. In: Receptor signal transduction protocols, vol. 259; 2004. p. 249-73.

26. Segawaa JSK. Constitutive exposure of phosphatidylserine on viable cell. PNAS. 2012;108(48):19246-51.

27. Piper RC. Endosomal Transportation via Ubiquitination. Trends Cell Biol. 2011;21(11):647-55.

28. Ehrlich WBM. Endocytosis by random initiation and stabilizatio of clathrincoated pits. Cell Press. 2004;118(5):591-605.

29. Nishimura NMT. Membrane re-modelling by BAR domain superfamily proteins via molecular and non-molecular factors. Biochem Soc Trans. 2018; 46(2):379-89.

30. Morel O, Jesel L, Freyssinet JM, Toti F. Cellular mechanisms underlying the formation of circulating microparticles. Arterioscler Thromb Vasc Biol. 2011; 31(1):15-26.

31. Gitz E, Pollitt AY, Gitz-Francois JJ, Alshehri O, Mori J, Montague S, Nash GB, Douglas MR, Gardiner EE, Andrews RK. CLEC-2 expression is maintained on activated platelets and on platelet microparticles. Blood. 2014;124(14):226270.

32. Badimon L, Suades R, Fuentes E, Palomo I, Padro T. Role of platelet-derived microvesicles as crosstalk mediators in Atherothrombosis and future pharmacology targets: a link between inflammation, atherosclerosis, and thrombosis. Front Pharmacol. 2016:7:293.

33. Flaumenhaft R, Dilks JR, Richardson J, Alden E, Patel-Hett SR, Battinelli E, Klement GL, Sola-Visner M, Italiano JE Jr. Megakaryocyte-derived microparticles: direct visualization and distinction from platelet-derived microparticles. Blood. 2009:113(5):1112-21.

34. Mitsios JV, Vini MP, Stengel D, Ninio E, Tselepis AD. Human platelets secrete the plasma type of platelet-activating factor acetylhydrolase primarily associated with microparticles. Arterioscler Thromb Vasc Biol. 2006;26(8):1907-13.

35. Lok CA, Nieuwland R, Sturk A, Hau CM, Boer K, Vanbavel E, Vanderpost JA. Microparticle-associated P-selectin reflects platelet activation in preeclampsia. Platelets. 2007;18(1):68-72.

36. Edelstein LC. The role of platelet microvesicles in intercellular communication. Platelets. 2017;28(3):222-7.

37. Boilard E, Duchez AC, Brisson A. The diversity of platelet microparticles. Curr Opin Hematol. 2015;22(5):437-44.

38. Platelet responsiveness and function during storage : implications for platelet transfusion therapy. Maastricht: Maastricht University; 2007.

39. Bhullar J, Bhopale VM, Yang M, Sethuraman K, Thom SR. Microparticle formation by platelets exposed to high gas pressures - an oxidative stress response. Free Radic Biol Med. 2016;101:154-62.

40. Ghasemzadeh M, Hosseini E, Roudsari ZO, Zadkhak P. Intraplatelet reactive oxygen species (ROS) correlate with the shedding of adhesive receptors, microvesiculation and platelet adhesion to collagen during storage: does endogenous ROS generation downregulate platelet adhesive function? Thromb Res. 2018;163:153-61.

41. Nomura MSS. Clinical significance of procoagulant microparticle. J Intensive Care. 2015;3(1):2

42. Morel $\mathrm{O}$, Toti F, Jesel $\mathrm{L}$, Freyssinet JM. Mechanisms of microparticle generation: on the trail of the mitochondrion! Semin Thromb Hemost. 2010; 36(8):833-44.

43. Smethurst PA. Aging of platelets stored for transfusion. Platelets. 2016;27(6): 526-34.

44. Goth CK, Halim A, Khetarpal SA, Rader DJ, Clausen H, Schjoldager KT. A systematic study of modulation of ADAM-mediated ectodomain shedding by site-specific O-glycosylation. Proc Natl Acad Sci U S A. 2015;112(47): 14623-8.

45. Arribas J, Borroto A. Protein ectodomain shedding. Chem Rev. 2002;102(12): 4627-38.

46. Au AE, Josefsson EC. Regulation of platelet membrane protein shedding in health and disease. Platelets. 2017;28(4):342-53.

47. Andrews RK, Gardiner EE. Metalloproteolytic receptor shedding...Platelets "acting their age". Platelets. 2016;27(6):512-8. 
48. Andrews RK, Karunakaran D, Gardiner EE, Berndt MC. Platelet receptor proteolysis: a mechanism for downregulating platelet reactivity. Arterioscler Thromb Vasc Biol. 2007;27(7):1511-20.

49. Quach ME, Chen W, Li R. Mechanisms of platelet clearance and translation to improve platelet storage. Blood. 2018;131(14):1512-21.

50. Jansen AJ, Josefsson EC, Rumjantseva V, Liu QP, Falet H, Bergmeier W, Cifuni SM, Sackstein R, von Andrian UH, Wagner DD, Hartwig JH, Hoffmeister KM. Desialylation accelerates platelet clearance after refrigeration and initiates GPIbalpha metalloproteinase-mediated cleavage in mice. Blood. 2012;119(5): 1263-73.

51. Coller BS, Kalomiris E, Steinberg M, Scudder LE. Evidence that glycocalicin circulates in normal plasma. J Clin Invest. 1984;73(3):794-9.

52. Bergmeier W, Piffath $\mathrm{CL}$, Cheng G, Dole VS, Zhang Y, von Andrian UH, Wagner DD. Tumor necrosis factor-alpha-converting enzyme (ADAM17) mediates GPIbalpha shedding from platelets in vitro and in vivo. Circ Res. 2004;95(7):677-83.

53. Aktas B, Pozgajova M, Bergmeier W, Sunnarborg S, Offermanns S, Lee D, Wagner DD, Nieswandt B. Aspirin induces platelet receptor shedding via ADAM17 (TACE). J Biol Chem. 2005;280(48):39716-22.

54. Hosseini E, Ghasemzadeh M, Nassaji F, Jamaat ZP. GPVI modulation during platelet activation and storage: its expression levels and ectodomain shedding compared to markers of platelet storage lesion. Platelets. 2017; 28(5):498-508.

55. Gardiner EE. Proteolytic processing of platelet receptors. Res Pract Thromb Haemost. 2018;2(2):240-50

56. Baaten CC, Swieringa F, Misztal T, Mastenbroek TG, Feijge MA, Bock PE, Donners MM, Collins PW, Li R, van der Meijden PE. Platelet heterogeneity in activation-induced glycoprotein shedding: functional effects. Blood advances. 2018;2(18):2320-31.

57. Gardiner E, Arthur J, Berndt M, Andrews R. Role of calmodulin in platelet receptor function. Curr Med Chem Cardiovasc Hematol Agents. 2005;3(4):283-7.

58. Hartmann $M$, Herrlich $A$, Herrlich P. Who decides when to cleave an ectodomain? Trends Biochem Sci. 2013;38(3):111-20.

59. Andrews RK, Munday AD, Mitchell CA, Berndt MC. Interaction of calmodulin with the cytoplasmic domain of the platelet membrane glycoprotein Ib-IX-V complex. Blood. 2001;98(3):681-7.

60. Wang Z, Li S, Shi Q, Yan R, Liu G, Dai K. Calmodulin antagonists induce platelet apoptosis. Thromb Res. 2010;125(4):340-50.

61. Stalker TJ, Newman DK, Ma P, Wannemacher KM, Brass LF. Platelet signaling. Handb Exp Pharmacol. 2012;210:59-85.

62. Arthur JF, Shen Y, Kahn ML, Berndt MC, Andrews RK, Gardiner EE. Ligand binding rapidly induces disulfide-dependent dimerization of glycoprotein VI on the platelet plasma membrane. J Biol Chem. 2007;282(42):30434-41.

63. Qiao J, Arthur JF, Gardiner EE, Andrews RK, Zeng L, Xu K. Regulation of platelet activation and thrombus formation by reactive oxygen species. Redox Biol. 2018;14:126-30.

64. Giannoni E, Buricchi F, Raugei G, Ramponi G, Chiarugi P. Intracellular reactive oxygen species activate Src tyrosine kinase during cell adhesion and anchorage-dependent cell growth. Mol Cell Biol. 2005;25(15):6391-403.

65. Yoo SK, Freisinger CM, LeBert DC, Huttenlocher A. Early redox, Src family kinase, and calcium signaling integrate wound responses and tissue regeneration in zebrafish. J Cell Biol. 2012;199(2):225-34.

66. Ray PD, Huang BW, Tsuji Y. Reactive oxygen species (ROS) homeostasis and redox regulation in cellular signaling. Cell Signal. 2012;24(5):981-90.

67. Brill A, Chauhan AK, Canault M, Walsh MT, Bergmeier W, Wagner DD. Oxidative stress activates ADAM17/TACE and induces its target receptor shedding in platelets in a p38-dependent fashion. Cardiovasc Res. 2009;84(1):137-44.

68. Yamamori T, Inanami O, Nagahata H, Cui Y-D, Kuwabara M. Roles of p38 MAPK, PKC and PI3-K in the signaling pathways of NADPH oxidase activation and phagocytosis in bovine polymorphonuclear leukocytes. FEBS Lett. 2000:467(2-3):253-8.

69. Bender M, Stegner D, Nieswandt B. Model systems for platelet receptor shedding. Platelets. 2017;28(4):325-32

70. Mondal NK, Chen Z, Trivedi JR, Sorensen EN, Pham SM, Slaughter MS, Griffith BP, Wu ZJ. Oxidative stress induced modulation of platelet integrin $\mathrm{a} 2 \mathrm{~b} \beta 3$ expression and shedding may predict the risk of major bleeding in heart failure patients supported by continuous flow left ventricular assist devices. Thromb Res. 2017;158:140-8.

71. Kulkarni S, Jackson SP. Platelet factor XIII and calpain negatively regulate integrin alphallbbeta3 adhesive function and thrombus growth. J Biol Chem. 2004;279(29):30697-706.
72. Wu Y, Asazuma N, Satoh K, Yatomi Y, Takafuta T, Berndt MC, Ozaki Y. Interaction between von Willebrand factor and glycoprotein lb activates Src kinase in human platelets: role of phosphoinositide 3-kinase. Blood. 2003; 101(9):3469-76.

73. Gardiner EE, Karunakaran D, Arthur JF, Mu FT, Powell MS, Baker RI, Hogarth PM, Kahn ML, Andrews RK, Berndt MC. Dual ITAM-mediated proteolytic pathways for irreversible inactivation of platelet receptors: de-ITAM-izing FcgammaRlla. Blood. 2008;111(1):165-74.

74. Cosentino-Gomes D, Rocco-Machado N, Meyer-Fernandes JR. Cell signaling through protein kinase C oxidation and activation. Int J Mol Sci. 2012;13(9): 10697-721.

75. Bridges $L C$, Bowditch RD. ADAM-integrin interactions: potential integrin regulated ectodomain shedding activity. Curr Pharm Des. 2005;11(7):837-47.

76. Yanez-Mo M, Sanchez-Madrid F, Cabanas C. Membrane proteases and tetraspanins. Biochem Soc Trans. 2011:39(2):541-6.

77. Jackson SP, Schoenwaelder SM. Procoagulant platelets: are they necrotic? Blood. 2010;116(12):2011-8.

78. Andrews RK, Gardiner EE. Basic mechanisms of platelet receptor shedding Platelets. 2017;28(4):319-24.

79. van Kruchten R, Mattheij NJ, Saunders C, Feijge MA, Swieringa F, Wolfs JL, Collins PW, Heemskerk JW, Bevers EM. Both TMEM16F-dependent and TMEM16F-independent pathways contribute to phosphatidylserine exposure in platelet apoptosis and platelet activation. Blood. 2013;121(10): 1850-7.

80. Rukoyatkina N, Mindukshev I, Walter U, Gambaryan S. Dual role of the p38 MAPK/CPLA2 pathway in the regulation of platelet apoptosis induced by ABT-737 and strong platelet agonists. Cell Death Dis. 2013;4:e931.

81. Chen Z, Mondal NK, Ding J, Gao J, Griffith BP, Wu ZJ. Shear-induced platelet receptor shedding by non-physiological high shear stress with short exposure time: glycoprotein Iba and glycoprotein VI. Thromb Res. 2015; 135(4):692-8.

82. Gardiner EE, Andrews RK. Platelet receptor expression and shedding: glycoprotein Ib-IX-V and glycoprotein VI. Transfus Med Rev. 2014;28(2):56-60.

83. Ghasemzadeh M, Hosseini E. Platelet granule release is associated with reactive oxygen species generation during platelet storage: a direct link between platelet pro-inflammatory and oxidation states. Thromb Res. 2017; 156:101-4.

84. Jilma-Stohlawetz P, Horvath M, Eichelberger B, Koren D, Jilma B, Panzer S. Platelet function under high-shear conditions from platelet concentrates. Transfusion. 2008;48(1):129-35.

85. Pennings GJ, Yong AS, Wong C, Al-Tamimi M, Gardiner EE, Andrews RK, Kritharides L. Circulating levels of soluble EMMPRIN (CD147) correlate with levels of soluble glycoprotein VI in human plasma. Platelets. 2014;25(8):639-42.

86. Naitoh K, Hosaka Y, Honda M, Ogawa K, Shirakawa K, Furusako S. Properties of soluble glycoprotein $\mathrm{VI}$, a potential platelet activation biomarker. Platelets. 2015;26(8):745-50.

87. Chen W, Liang X, Syed AK, Jessup P, Church WR, Ware J, Josephson CD, Li R. Inhibiting GPlba shedding preserves post-transfusion recovery and hemostatic function of platelets after prolonged StorageHighlights. Arterioscler Thromb Vasc Biol. 2016;36(9):1821-8.

88. Boomgaard MN, Gouwerok CW, Homburg CH, de Groot G, MJ IJ, de Korte D. The platelet adhesion capacity to subendothelial matrix and collagen in a flow model during storage of platelet concentrates for 7 days. Thromb Haemost. 1994;72(4):611-6.

89. Hosseini E, Beshkar P, Ghasemzadeh M. Reverse correlations of collagendependent platelet aggregation and adhesion with GPVI shedding during storage. J Thromb Thrombolysis. 2018:46(4):534-40.

90. Aubron C, Flint AW, Ozier Y, McQuilten Z. Platelet storage duration and its clinical and transfusion outcomes: a systematic review. Crit Care. 2018;22(1):185.

91. Ng MS, Tung JP, Fraser JF. Platelet storage lesions: what more do we know now?. Transfusion medicine reviews. 2018;32(3):144-54

92. Soslau G, Giles J. The loss of sialic acid and its prevention in stored human platelets. Thromb Res. 1982;26(6):443-55.

93. Bergmeier W, Burger PC, Piffath CL, Hoffmeister KM, Hartwig JH, Nieswandt B, Wagner DD. Metalloproteinase inhibitors improve the recovery and hemostatic function of in vitro-aged or-injured mouse platelets. Blood. 2003;102(12):4229-35.

\section{Publisher's Note}

Springer Nature remains neutral with regard to jurisdictional claims in published maps and institutional affiliations. 